Repository of the Max Delbrück Center for Molecular Medicine (MDC) Berlin (Germany)

\title{
Combining photonic crystal and optical Monte Carlo simulations: implementation, validation and application in a positron emission tomography detector.
}

Thalhammer, C., Breuer, J., Fuehrer, T., Popescu, A., Hedler, H., Walther, T., Niendorf, T.

This is the accepted version of the article, i.e. the version that has been revised by the author to incorporate review suggestions and that has been accepted by IEEE for publication. The article was published in final edited form as:

IEEE Transactions on Nuclear Science. 2014 Dec ; 61(6): 3618-3626

doi: 10.1109/TNS.2014.2365879

\section{IEEE $>$}

(C) 2014 IEEE. Personal use of this material is permitted. Permission from IEEE must be obtained for all other uses, in any current or future media, including reprinting/republishing this material for advertising or promotional purposes, creating new collective works, for resale or redistribution to servers or lists, or reuse of any copyrighted component of this work in other works. 


\title{
Combining Photonic Crystal and Optical Monte Carlo Simulations: Implementation, Validation and Application in a Positron Emission Tomography Detector
}

\author{
Christof Thalhammer, Johannes Breuer, Thorsten Führer, Alexandru Popescu, Harry Hedler, Thomas Walther, \\ and Thoralf Niendorf
}

\begin{abstract}
This paper presents a novel approach towards incorporating photonic crystals (PhCs) into optical Monte Carlo (MC) simulations. This approach affords modeling the full diffractive nature of PhCs including their reflection and transmission behavior as well as the manipulation of the photon trajectories through light scattering. The main purpose of this tool is to study the impact of PhCs on the light yield and timing performance of scintillator-based detectors for positron emission tomography (PET). To this end, the PhCs are translated into look-up tables and implemented into the optical MC algorithm. Our simulations are validated in optical experiments using PhC samples fabricated with electron beam lithography. The experimental results indicate that the simulations match the measurements within the accuracy of the experiments. The application of the combined simulation technique to a PET detector module predicts an increase of the total light yield by up to $23 \%$ for $\mathrm{PhC}$ coatings versus the reference without PhCs. Timing calculations reveal an improvement of the coincident resolving time by up to $6 \%$. The results underline the potential of $\mathrm{PhCs}$ to improve light yield and timing of PET detector modules.
\end{abstract}

Index Terms-Positron emission tomography (PET), Photonic Crystals, Monte Carlo simulations, coincident resolving time, timing resolution, PET detector.

\section{INTRODUCTION}

D ETAILING the propagation and extraction of light in scintillator-based detectors used for positron emission tomography (PET) is beneficial to understand and enhance the total amount of light reaching the photosensor and the coincidence resolving time (CRT) [1]-[5]. Energy resolution and CRT are limited by insufficient light extraction from the scintillators [6]. If the photons are reflected many times from the scintillator exit face, the path length inside the scintillator

This work has received funding from Siemens Medical Solutions.

C. Thalhammer is with the Berlin Ultrahigh Field Facility (B.U.F.F.), MaxDelbrueck Center for Molecular Medicine, D-12125 Berlin, Germany, and also with Siemens Corporate Technology, D-81739 München, Germany (email: christof.thalhammer@mdc-berlin.de).

J. Breuer is with Siemens Healthcare, D-91301 Forchheim, Germany (email: johannes.breuer@siemens.com).

T. Führer and T. Walther are with the Technical University Darmstadt, D64289 Darmstadt, Germany (e-mail: thorsten.fuehrer@physik.tu-darmstadt.de; thomas.walther@physik.tu-darmstadt.de).

A. Popescu and H. Hedler are with Siemens Corporate Technology, D81739 München, Germany (e-mail: alexandru.popescu@ siemens.com; harry. hedler@siemens.com).

T. Niendorf is with the Berlin Ultrahigh Field Facility (B.U.F.F.), MaxDelbrueck Center for Molecular Medicine, D-12125 Berlin, Germany (e-mail: thoralf.niendorf@mdc-berlin.de). is prolonged, hence increasing absorption losses. An efficient extraction of photons at their first incidence on the exit face is expected to improve the CRT because the timing is not exclusively defined by the total amount of detected photons, but also depends on their propagation time distribution [7]. Currently, photon extraction is limited by light trapping inside the scintillator due to total internal reflection (TIR) since common inorganic scintillators such as lutetium oxyorthosilicate (LSO) have a high refractive index $\left(n_{\text {LSO }}=1.82\right)$ versus the optical glue $\left(n_{\text {Glue }} \approx 1.5\right.$ ) used to couple the scintillator and the photosensor [8]. Thin layers of photonic crystals $(\mathrm{PhCs})$ bear the potential to reduce light trapping and increase the extraction efficiency from high index media [9]. PhC structures exhibit a periodically modulated refractive index with characteristic dimensions in the range of the wavelength of the incident light, thus acting like a diffraction grating that scatters impinging photons into various diffraction orders [10]. This effect can lead to the extraction of photons with shallow incident angles beyond the TIR threshold. First applications of $\mathrm{PhC}$ technology to inorganic scintillators showed improved light output and timing resolution for individual LSO crystals [7], [11].

Monte Carlo (MC) simulations are a powerful tool to study the propagation of scintillation photons in PET detectors using ray tracing algorithms based on geometric optics [1]-[3], [12]. Since these techniques are not capable of computing the diffractive effects of PhCs, an additional tool is required to solve Maxwell's equations. For the combination of these two techniques, analytical models [8] or look-up tables containing the transmission coefficients with respect to the incident angle [6] were proposed. Apart from exhibiting a distinct transmission behavior, $\mathrm{PhCs}$ also manipulate the photon trajectories through light scattering into various diffraction orders which presents a limitation of previous approaches. Realizing this challenge, this work proposes the implementation of $\mathrm{PhCs}$ into optical MC simulations. This combined approach allows modeling the full diffractive nature of PhCs including their transmission characteristics and light scattering properties. The technique is validated in angle-resolved transmission measurements using $\mathrm{PhC}$ samples fabricated with electron beam lithography (EBL). The approach is then applied to study the impact of an optimized PhC configuration on the light yield and CRT of a state-of-the-art PET detector module. 


\section{Materials And Methods}

\section{A. Implementation of PhCs into Optical Simulations}

All optical MC simulations were conducted with the commercial software Zemax (Radiant Zemax, Redmond, WA, USA). To calculate the diffractive behavior of $\mathrm{PhCs}$, GDCalc (KJ Innovation, Santa Clara, CA, USA) was used which is based on the rigorous coupled-wave analysis [13]. In this approach, the $\mathrm{PhC}$ geometry is represented by a stack of layers that contain cuboids of a certain refractive index forming a periodic pattern (see Fig. 1). The algorithm solves Maxwell's equations using a Fourier expansion of the electric field and yields scattering matrices $\boldsymbol{R}$ and $\boldsymbol{T}$. These matrices correlate the amplitudes and phases of the incident and diffracted electromagnetic waves and facilitate the calculation of the diffraction order efficiencies for any given polarization of the incident electromagnetic wave [14].

For the implementation, $\mathrm{PhCs}$ were translated into look-up tables (LUTs) that contain bins of incident angles $\theta$ and $\phi$ (see Fig. 1). The elevation angle $\theta$ ranges from $0^{\circ}$ to $180^{\circ}$ with $\theta$-values larger than $90^{\circ}$ corresponding to photons that impinge on the $\mathrm{PhC}$ from the optical glue side instead of the scintillator side. Due to the symmetry of the $\mathrm{PhC}$, the azimuth angle $\phi$ was varied from $0^{\circ}$ to $30^{\circ}$ for hexagonal and from $0^{\circ}$ to $45^{\circ}$ for orthogonal $\mathrm{PhC}$ gratings. For each pair of $\theta$ and $\phi$, the scattering matrices and wave vectors of all non-evanescent reflected and transmitted orders were calculated with GD-Calc and written into the LUT. To take into account the wavelength-dependent behavior of PhCs, this procedure was repeated for each wavelength of the spectrum of interest yielding a series of LUTs. The LUTs were included into a custom procedure based on a dynamic link library (DLL) written in the programming language $\mathrm{C}$. The $\mathrm{PhC}$ coating was implemented into Zemax by assigning the DLL to a certain material interface in the ray tracing tool. For each photon hitting this interface, Zemax passes the direction cosines, polarization, and wavelength of the incident photon to the DLL, which utilizes this information in conjunction with the LUT entries to calculate the diffraction efficiencies. Interpreting these diffraction efficiencies as MC probabilities, a random number is used to decide between reflection and transmission and to select a certain order. Finally, the photon is returned to Zemax with the diffracted wave vector $\boldsymbol{k}$ ' taken from the LUT for the selected order (see Fig. 2).

\section{B. Experimental Validation of Transmission Characteristics}

1) PhC Fabrication: $\mathrm{PhC}$ samples were fabricated with hexagonal and orthogonal grating symmetry. The bulk material of the PhCs was a $450 \mathrm{~nm}$ thick layer of electron beam resist ( $n_{\text {Resist }}=1.59$ at wavelength $\lambda=405 \mathrm{~nm}$ ), which was spincoated on a glass substrate (Borofloat, $n_{\mathrm{BF}}=1.48$ at $\lambda=$ $405 \mathrm{~nm})$. The $\mathrm{PhC}$ structures were written directly into the resist layer by electron beam lithography (EBL) with a square shaped beam followed by wet development. The patterned area was $8 \mathrm{~mm} \times 8 \mathrm{~mm}$ per sample. Using EBL requires a conducting substrate to avoid charging effects. For this purpose, magnetron sputtering was used to deposit a $100 \mathrm{~nm}$ thick layer of tin-doped indium oxide (ITO, $n_{\mathrm{ITO}}=2.06$ at

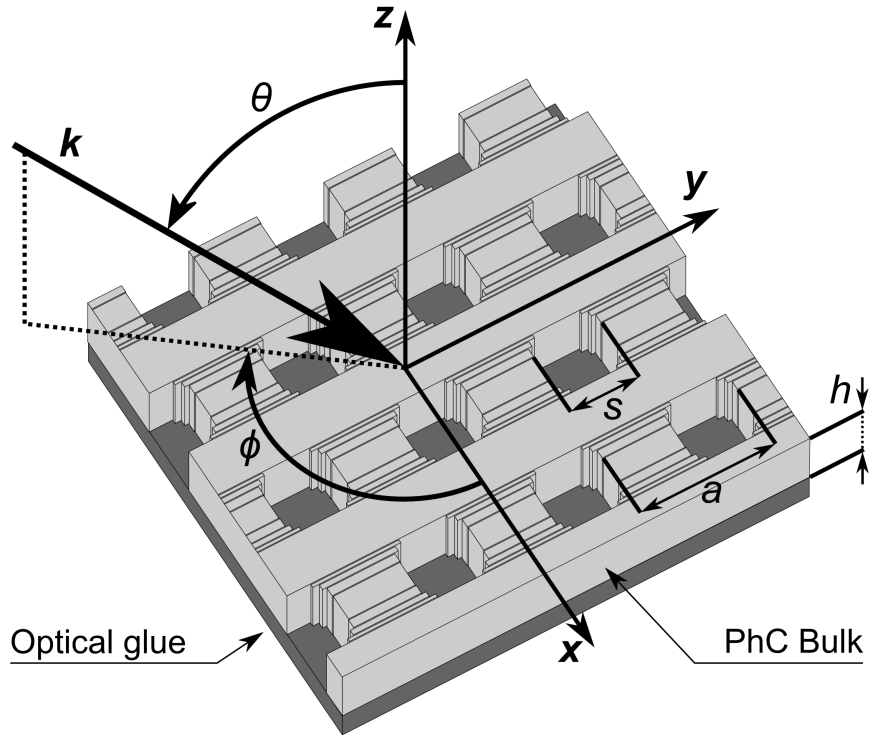

Fig. 1. Schematic representation of a PhC coating within GD-Calc. The example depicts a hexagonal array of cavities. The rounded cavities are approximated through a series of rectangular cuboids. The geometry of the $\mathrm{PhC}$ is defined through its pitch $a$, the cavity size $s$, and its height $h$. The incident angles $\theta$ and $\phi$ of an incident photon with a wave vector $\boldsymbol{k}$ are defined with respect to the local coordinate system $(\boldsymbol{x}, \boldsymbol{y}, \boldsymbol{z})$ of the PhC. The number of cuboids approximating the rounded edges has been reduced and the medium above the $\mathrm{PhC}$ as well as the filling material of the cavities are not shown for illustration purposes.

$\lambda=405 \mathrm{~nm}$ ) on the glass substrate prior to spin-coating of the resist. Finally, the glass wafer was cut into individual chips using a dicing saw. The geometric parameters $a$ and $s$ (see Fig. 1) were chosen based on preliminary simulations with the goal to obtain diverse transmission characteristics with pronounced diffractive effects. To measure the actual geometric parameters of the fabricated $\mathrm{PhCs}$, scanning electron microscopy (SEM) images were acquired.

2) Transmission Measurements: The angular transmission characteristics of the samples were measured with the experimental setup shown in Fig. 3a. In this setup, the samples were positioned in a holder providing a distance of $200 \mu \mathrm{m}$ to a bare silicon photodiode (PD) with a sensitive area of $10 \mathrm{~mm} \times 10 \mathrm{~mm}$. The orientation of the samples was chosen so that the PhC coating was facing the sensor (see Fig. 3b). The sample was illuminated under various incident angles through a glass hemisphere $\left(n_{\mathrm{HS}}=1.82\right.$ at $\left.\lambda=405 \mathrm{~nm}\right)$ placed on top. A $700 \mu \mathrm{m}$ thick film of Polydimethylsiloxan (PDMS, $n_{\text {PDMS }}=1.45$ at $\lambda=405 \mathrm{~nm}$ ) was used as optical coupling agent between sample and hemisphere. The sample holder was placed in a motorized goniometer which allowed the variation of the azimuth angle $\phi_{\mathrm{L}}$. A laser diode module was mounted on the arm of the goniometer to vary the elevation angle $\theta_{\mathrm{L}}$ of the light beam. The laser had a wavelength of $405 \mathrm{~nm}$ and was equipped with lenses and an aperture to provide a collimated beam with a Gaussian profile. Its full width at half maximum is $\mathrm{FWHM}=400 \mu \mathrm{m}$. The polarization of the beam was adjusted using a half-wave plate. The lateral position of the sample and the PD was chosen off-center of the goniometer to account for the lateral displacement of the laser beam for $\theta_{\mathrm{L}}>0^{\circ}$ 


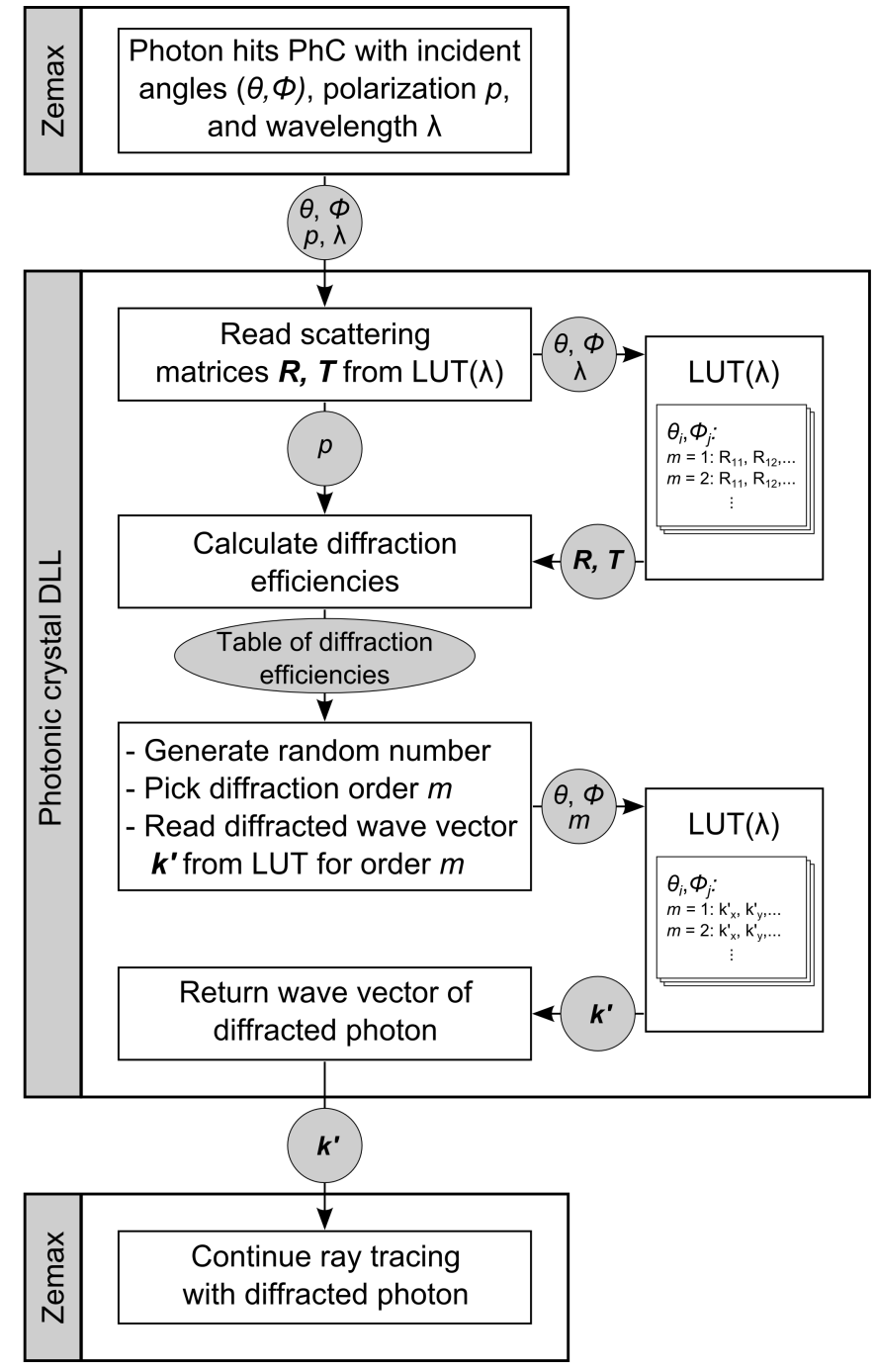

Fig. 2. Schematic illustration of the custom-written DLL and its implementation into the ray tracing algorithm Zemax.

(see Fig. 3b). The incident angle $\theta_{\text {Sample }}$ on the sample/air interface and the elevation angle $\theta_{\mathrm{L}}$ of the laser beam are correlated by Snell's law via $\theta_{\text {Sample }}=\operatorname{asin}\left[\sin \left(\theta_{\mathrm{L}}\right) \cdot n_{\mathrm{HS}} / n_{\mathrm{BF}}\right]$. This allows sweeping $\theta_{\text {Sample }}$ from $0^{\circ}$ to $90^{\circ}$ using a range for $\theta_{\mathrm{L}}$ of $0^{\circ}$ to $54.4^{\circ}$. A custom-written program controlled the goniometer movement and the laser power. The PD current was converted into voltage by a transimpedance amplifier and recorded by a computer equipped with an analog-todigital converter as $U_{\text {Meas }}\left(\theta_{\mathrm{L}}, \phi_{\mathrm{L}}, p\right)$, where $p$ identifies the polarization state of the laser beam. The elevation angle $\theta_{\mathrm{L}}$ was varied from $0^{\circ}$ to $60^{\circ}$ in $0.5^{\circ}$-increments, the azimuth angle $\phi_{\mathrm{L}}$ ranged from $0^{\circ}$ to $90^{\circ}$ with $1^{\circ}$-increments. For each incident angle, measurements were performed using $\mathrm{S}$ - and $\mathrm{P}$ polarization (electric field vector perpendicular or parallel to the plane of incidence). Each measurement was repeated 50 times with an individual duration of $28.5 \mathrm{~ms}$ at a laser output power of $5 \mathrm{~mW}$, which led to a standard deviation below $0.1 \%$.

3) Transmission Simulations: The experimental setup was simulated with the ray tracing tool. The profile of the laser beam was measured using a charge-coupled device camera and

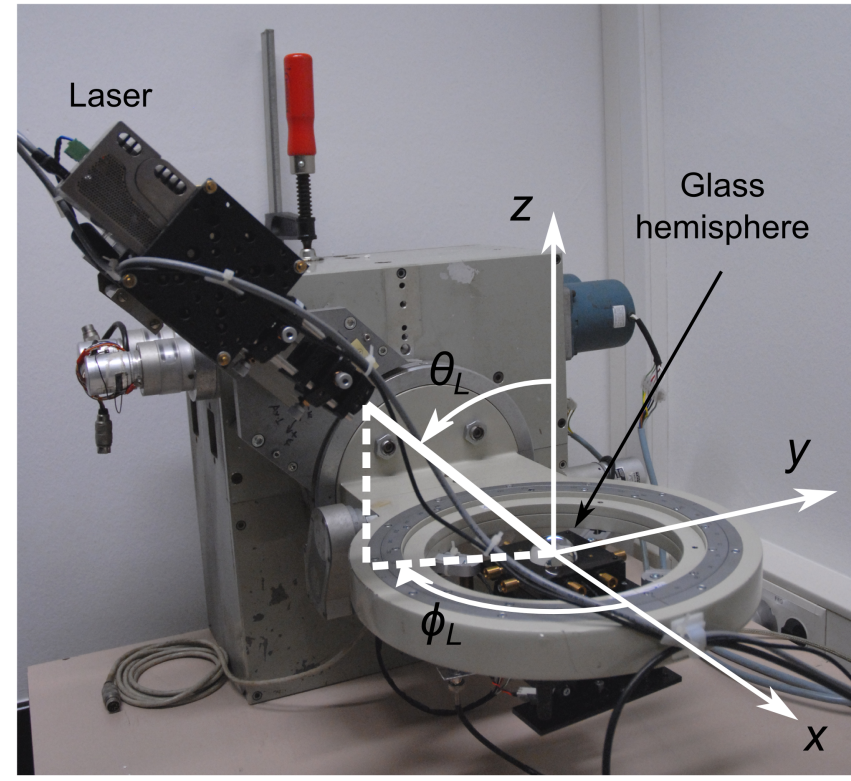

(a)

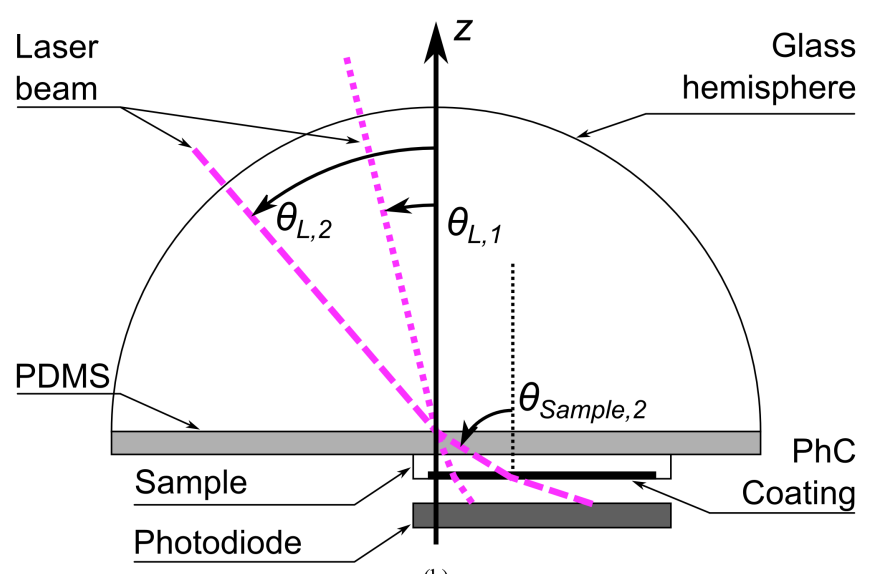

(b)

Fig. 3. (a) Photograph of the goniometer setup. The white line illustrates a laser beam incident on the hemisphere with angles $\theta_{\mathrm{L}}$ and $\phi_{\mathrm{L}}$. (b) Schematic showing the arrangement of PD, sample, PDMS film and glass hemisphere. Sample and PD are placed off-center because of the lateral displacement of the laser beam. The violet lines depict laser beams for two different incident angles $\theta_{\mathrm{L}, 1}$ and $\theta_{\mathrm{L}, 2}$ and illustrate the lateral shift of the beam on the PD. Due to refraction, the incident angle of the laser on the $\mathrm{PhC}$ coating $\theta_{\text {Sample, } 2}$ is larger than $\theta_{\mathrm{L}, 2}$

used to model a light source in the optical simulations. The polarization- and angle-dependent sensitivity of the photodiode was measured using the goniometer setup without sample and hemisphere. This characteristic was also implemented into the simulation model. Since scattering from impurities caused fluctuations in $U_{\text {Meas }}$ at some $\theta_{\mathrm{L}}$-values, the average measured signal $U_{\text {Meas }}$ for $\theta_{\mathrm{L}}<3^{\circ}$ was used to scale the simulated data instead of using only $\theta_{\mathrm{L}}=0^{\circ}$. To estimate the accuracy of the computational model, a calibration experiment was conducted using a sample without $\mathrm{PhC}$ coating. The relative difference between simulated and measured data was averaged over all angles $\theta_{\mathrm{L}}, \phi_{\mathrm{L}}$ and the two polarization states $\mathrm{S}$ and $\mathrm{P}$. This value was used as the systematic uncertainty $\gamma_{\text {Simu }}$ for the simulated voltage $U_{\text {Simu }}$. This error of the simulations covers impurities of the sample, interfaces that were not perfectly 
coplanar and light scattering through electrical connections or mechanical fixations. The statistical error of the MC simulations was estimated through the standard deviation of the results from 10 repeated computations. It was found to be less than $0.1 \%$, since $10^{5}$ photons were simulated per incident angle and polarization state. The calibration experiment was also used to estimate the accuracy of the goniometer setup $\Delta \theta_{\mathrm{L}}$ regarding the calibration of $\theta_{\mathrm{L}}$. For this purpose the TIR-related cutoff was located in the measured PD signal and compared to the position in the simulated signal with its intrinsically perfect accuracy in $\theta_{\mathrm{L}}$. To evaluate $\Delta \phi_{\mathrm{L}}$, i.e. the precision of the experiment regarding $\phi_{\mathrm{L}}$, the locations of certain periodic features in measured and simulated $\mathrm{PhC}$ transmission characteristics were compared. Finally, the uncertainty $\gamma_{\text {Meas }}$ of the measured PD signal was determined by analyzing the variations of $U_{\text {Meas }}$ for changes $\pm \Delta \theta_{\mathrm{L}}$ and $\pm \Delta \phi_{\mathrm{L}}$. The results of this analysis are given in section III-B2. The agreement between $U_{\text {Meas }}$ and $U_{\text {Simu }}$ was analyzed using a two-sample Kolmogorov-Smirnov (KS) test [15]. In this procedure, a p-value is calculated based on the differences of two continuous distributions. The null hypothesis $H_{0}$, which states that the two functions stem from the same distribution, is rejected if the derived p-value is lower than the significance level $\alpha$. In this study, a KS test was conducted for all samples comparing the one-dimensional data sets $U_{\text {Meas }}\left(\theta_{\mathrm{L}}\right)$ and $U_{\text {Simu }}\left(\theta_{\mathrm{L}}\right)$ for fixed values of $\phi_{\mathrm{L}}$ and $p$ at a significance level $\alpha=0.05$. The results of this analysis are summarized in the agreement metric $\eta$, which is defined as the number of one-dimensional data sets for which $H_{0}$ was not rejected, divided by the total amount of data sets per sample.

\section{Simulation Study of the Impact of PhCs on the Performance of a State-of-the-Art PET Detector}

1) Detector Setup: The simulated PET detector consists of an array of $8 \times 8$ LSO scintillator crystals of the size $4 \mathrm{~mm} \times$ $4 \mathrm{~mm} \times 20 \mathrm{~mm}$ and a refractive index of $n_{\text {LSO }}=1.82$ [12], [16] as depicted in Fig. 4. The LSO pixels were modeled as polished crystals with a Gaussian surface scattering coefficient of $0.02 \mathrm{rad}$. Bulk absorption and scattering are characterized by mean free paths of $600 \mathrm{~mm}$ and $250 \mathrm{~mm}$ [12]. The scintillator pixels are closely packed and separated from each other by thin air gaps. Due to limitations of the reflection models at rough surfaces in Zemax, these air gaps were chosen to be only $50 \mathrm{~nm}$ to approximate light transmission behavior from pixel to pixel. The array is surrounded on 5 sides by a $20 \mu \mathrm{m}$ layer of air and specular reflective wrapping which has a reflection, transmission and absorption coefficient of $92 \%$, $3 \%$, and $5 \%$, respectively. As for the pixel spacing, these parameters were chosen to match experimental results. On the bottom, the LSO array is coupled to an $8 \times 8$ array of silicon photomultipliers (SiPM) [17], [18] via a $100 \mu \mathrm{m}$ layer of optical glue $\left(n_{\text {Glue }}=1.47\right)$ [12]. The SiPMs are modeled as bulk silicon material with a refractive index of 5.06 [19], which is encapsulated with a $300 \mu \mathrm{m}$ thick epoxy window $\left(n_{\text {Epoxy }}=1.59\right)$ [20]. The SiPMs are equipped with a single layer antireflective coating based on a quarter wave film for $420 \mathrm{~nm}$ with a thickness of $37 \mathrm{~nm}$ and a refractive index of

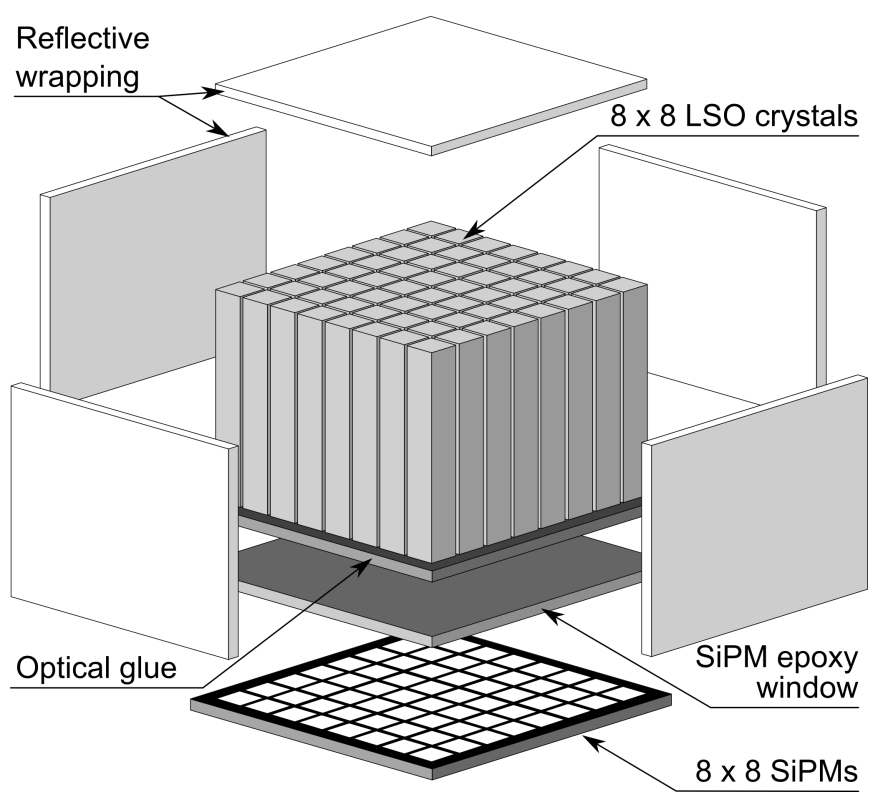

Fig. 4. Schematic view of the PET detector module used in the simulation study. The module includes an $8 \times 8$ array of LSO pixels, reflective wrapping, optical glue, and an array of $8 \times 8 \mathrm{SiPMs}$ with an epoxy window. (Dimensions are not to scale)

$n_{\text {Layer }}=2.84$, derived from the geometric mean of the silicon and epoxy refractive indices. Each sensor has a geometric fill factor of $64 \%$. This detector setup is similar to current stateof-the-art PET detector modules in terms of block size and LSO pixel dimensions [21], [22].

2) Optical Simulations: The software Geant4 [23] was used to calculate the interaction of gamma photons with the scintillator crystals (absorption length $=12 \mathrm{~mm}$ [16]). For this, a monoenergetic gamma source was simulated, which produced $511 \mathrm{keV}$ photons that were uniformly distributed over the entrance face of the LSO crystals and entered the scintillators perpendicularly. The locations of energy deposition of 4000 gamma photons were listed and transferred to Zemax as coordinates of the scintillation light sources. The scintillation light is assumed to be emitted isotropic and randomly polarized. As explained above, the PhC DLL analyzes the polarization of each photon to calculate the diffraction efficiencies. Hence, the incidence of randomly polarized photons onto a $\mathrm{PhC}$ leads to a transmission behavior that corresponds to the average of the S- and P-polarized $\mathrm{PhC}$ characteristics. The optical simulations in Zemax used a spectral distribution of the generated scintillation photons derived from sampling the LSO emission spectrum presented in [16] in $20 \mathrm{~nm}$ intervals from $380 \mathrm{~nm}$ to $600 \mathrm{~nm}$. To pronounce the impact of the wavelength-dependent $\mathrm{PhC}$ behavior on the light yield and timing, dispersion effects of the optical properties of the detector components were neglected with the exception of the SiPMs. These were modeled to have a photon detection efficiency (PDE) as described by the vendor [20] with values ranging from $25 \%$ at $\lambda=600 \mathrm{~nm}$ up to $35 \%$ at $\lambda=420 \mathrm{~nm}$. With $4 \cdot 10^{6}$ simulated scintillation photons the statistical error for the light yield, i.e. the fraction of all simulated photons reaching a photosensor, was below $0.1 \%$. The results from 
the MC computations were post-processed with Matlab (The Mathworks, Natick, MA, USA).

3) Timing Simulations: The timing resolution was estimated with a MC algorithm introduced by Breuer et al. [24], that uses the results from the optical simulations. This technique takes into account specific SiPM characteristics such as optical crosstalk, dark counts and afterpulsing. Also, the amplifier electronics and a leading edge discriminator are simulated in order to derive the CRT of the detector. For these simulations, data of a commercially available SiPM was used [20], with a cross talk probability of $27 \%$, a dark count rate of $100 \mathrm{kcps} / \mathrm{mm}^{2}$, an afterpulsing probability of $40 \%$, an overvoltage of $2.5 \mathrm{~V}$, a peak PDE of $35 \%$, and a single photon timing resolution of 230 ps. For each detector configuration, the discriminator threshold was optimized to obtain the minimum possible CRT. The timing simulations used $10^{5}$ gamma events, which resulted in a statistical error of the CRT below $0.5 \mathrm{ps}$.

4) Optimization of PhCs: The transmission characteristic of a PhC is influenced by its geometry and the refractive indices of its constituents. Together with the angular distribution of photons incident on the $\mathrm{PhC}$, it defines the extraction efficiency which is the fraction of the extracted light relative to the number of incident photons. The angular distribution derived from the optical simulations for the reference setup was used in conjunction with a sweep of the geometry parameters $a, s$ and $h$ (see Fig. 1) to obtain optimized PhC configurations with a high extraction efficiency. Preliminary simulations and the results presented in [8], [25] show that a bulk material with a high refractive index and strong index modulation within the $\mathrm{PhC}$ yields the strongest diffraction effects. Hence, the PhC was modeled to consist of a high index polymer $\left(n_{\text {Polymer }}=\right.$ 2.09 at $\lambda=420 \mathrm{~nm}$ [26]) with air cavities.

\section{RESUlTS AND Discussion}

\section{A. Implementation of PhCs into Optical Simulations}

For each $\mathrm{PhC}$ configuration, the corresponding LUTs were calculated prior to their implementation. The maximum number of $\left(\theta_{\mathrm{L}}, \phi_{\mathrm{L}}\right)$-pairs was 16200 per wavelength $\left(\theta_{\mathrm{L}}\right.$ from $0^{\circ}$ to $180^{\circ}$ with $0.5^{\circ}$ increment; $\phi_{\mathrm{L}}$ from $0^{\circ}$ to $45^{\circ}$ with $1^{\circ}$ increment). This required a mean computation time of $45 \mathrm{~s}$ per $\left(\theta_{\mathrm{L}}, \phi_{\mathrm{L}}\right)$-pair using a computer equipped with a dual core processor (Intel Core2Duo E8400, $3 \mathrm{GHz}$ ). The implementation of the PhC DLL into the optical simulations causes an increase in the ray tracing computation time by up to $50 \%$ per invocation. The impact on the total simulation time for more complex models strongly depends on the geometry, the number of photon-PhC interactions, and the lengths of the individual photon trajectories. In this work, the simulation time for the PET detector module was reduced by up to $30 \%$ for configurations using $\mathrm{PhCs}$ due to attenuated light trapping which led to shorter photon paths.

\section{B. Experimental Validation of Transmission Characteristics}

1) PhC Fabrication: Five different $\mathrm{PhC}$ samples were fabricated, two with a hexagonal and three with an orthogonal grating symmetry. A summary of the specified geometry
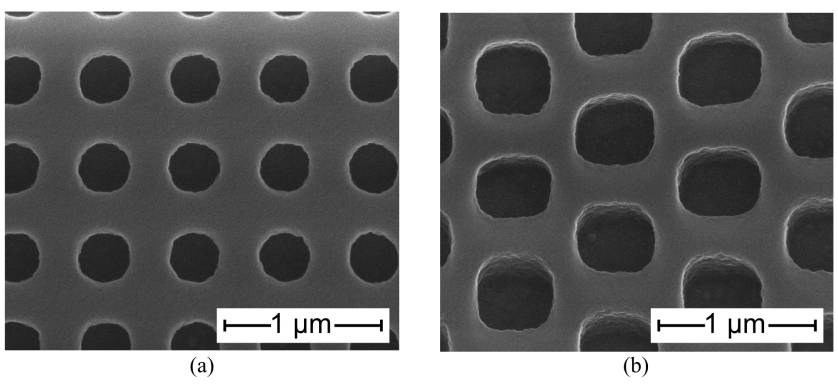

Fig. 5. SEM images of PhC samples $\mathrm{O} 2$ (a) and $\mathrm{H} 1$ (b) written into the electron beam resist. The images illustrate the rounding of the cavities due to the misalignment of the electron beam focus. The image in (a) was taken from a perpendicular perspective, whereas the perspective is tilted by $20^{\circ}$ in (b).

TABLE I

SUMMARY OF SAMPLE CHARACTERISTICS: SPECIFIED (SUBSCRIPT "SPEC") AND MEASURED (SUBSCRIPT "MEAS") GEOMETRY PARAMETERS AND AGREEMENT METRIC $\eta$ DERIVED FROM THE COMPARISON OF SIMULATED AND MEASURED TRANSMISSION DATA.

\begin{tabular}{|c|c|c|c|c|c|c|}
\hline $\begin{array}{c}\text { Sample } \\
\text { name }\end{array}$ & $\begin{array}{l}\text { Grating } \\
\text { symmetry }\end{array}$ & $\begin{array}{l}a_{\text {Spec }} \\
\text { in } \mathrm{nm}\end{array}$ & $\begin{array}{l}a_{\text {Meas }} \\
\text { in } \mathrm{nm}\end{array}$ & $\begin{array}{l}s_{\text {Spec }} \\
\text { in } \mathrm{nm}\end{array}$ & $\begin{array}{l}s_{\text {Meas }} \\
\text { in } \mathrm{nm}\end{array}$ & $\begin{array}{l}\text { Agreement } \\
\text { metric } \eta\end{array}$ \\
\hline H1 & Hex. & 798 & $793 \pm 4$ & 463 & $506 \pm 20$ & $71 \%$ \\
\hline $\mathrm{H} 2$ & Hex. & 630 & $633 \pm 4$ & 277 & $277 \pm 10$ & $98 \%$ \\
\hline O1 & Orth. & 672 & $670 \pm 6$ & 325 & $379 \pm 10$ & $73 \%$ \\
\hline $\mathrm{O} 2$ & Orth. & 588 & $584 \pm 7$ & 282 & $325 \pm 12$ & $71 \%$ \\
\hline $\mathrm{O} 3$ & Orth. & 504 & $504 \pm 3$ & 290 & $299 \pm 9$ & $77 \%$ \\
\hline
\end{tabular}

parameters and their values confirmed with SEM is surveyed in Table I. The measured values for the pitch $a$ differ from the specifications by $3 \%$ or less. The cavity size $s$ deviates by up to $17 \%(\mathrm{O} 1)$, which is significantly larger than the expected accuracy of a few nanometers. This stems from inaccuracies in the adjustment of the EBL focus plane, which were caused by the transparency of the substrate. For the same reason, the cavities shown in the SEM images in Fig. 5 have a rather round geometry although a quadratic electron beam was used. Variations of the $\mathrm{PhC}$ bulk thickness $h$ were negligible.

2) Comparison between Transmission Measurements and Simulations: The calibration using a sample without $\mathrm{PhC}$ coating indicates that the relative difference between measured and simulated PD signals varies only weakly with $\phi_{\mathrm{L}}$ and the polarization. The largest discrepancies are observed at $\theta_{\mathrm{L}^{-}}$ values close to the TIR-cutoff. In this region, the division by very small PD voltages can lead to relative differences of up to $90 \%$. The mean difference is $7 \% \pm 1 \%$, which leads to an uncertainty of the simulations $\gamma_{\text {Simu }}$ of $8 \%$. Fig. 6 shows the comparison of the measured and simulated calibration experiment for S- and P-polarization. Since the sample had no $\mathrm{PhC}$ coating, TIR was expected to occur at $\theta_{\mathrm{L}}=33^{\circ}$ which is the critical angle for the extraction of light from the glass hemisphere into air. This is clearly exemplified by the sharp cutoff in the PD voltage curve shown in Fig. 6.

After this calibration, transmission measurements of the $\mathrm{PhC}$ samples were performed. The LUTs corresponding to the $\mathrm{PhC}$ samples were calculated for a single wavelength of $405 \mathrm{~nm}$ using the geometry parameters from the SEM investigations. These computations also took into account the presence of the ITO layer between substrate and resist, which 


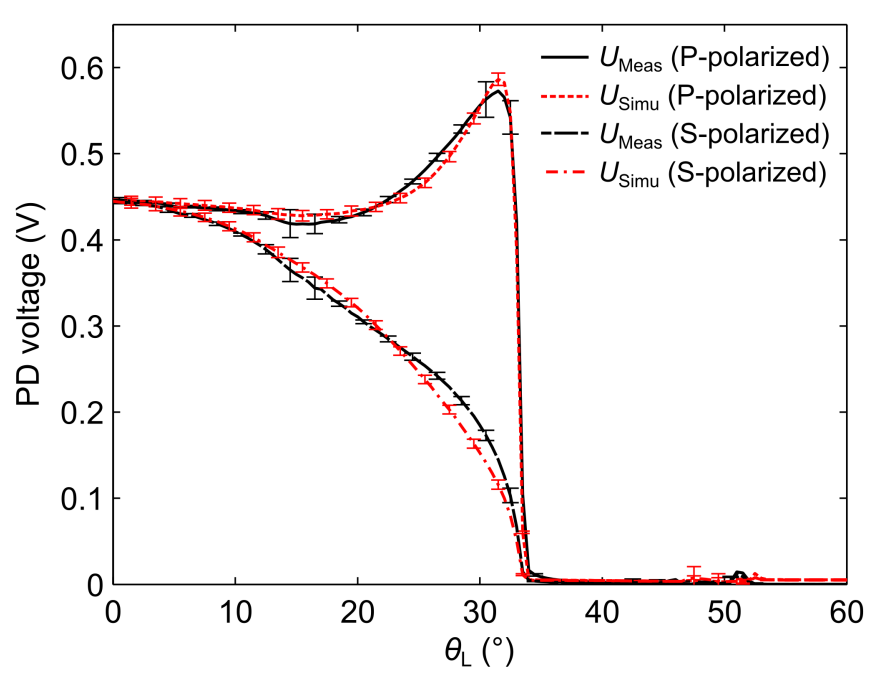

Fig. 6. PD voltage versus elevation angle $\theta_{\mathrm{L}}$ averaged over all azimuth angles $\phi_{\mathrm{L}}$, for a calibration experiment using an unstructured sample. The mean difference between measured and simulated data serves to estimate the accuracy of the computational model. The effect of TIR is clearly visible at $\theta_{\mathrm{L}}$ $=33^{\circ}$. The curves represent all acquired data points. Error bars are displayed at selected points.

was found to have only minor impact on the transmission properties of the samples. Subsequently, the samples were included into the MC simulations using the DLL-based approach. The periodic transmission behavior of the $\mathrm{PhC}$ samples was used to determine $\Delta \phi_{\mathrm{L}}$. The data acquired with a sampling increment for $\phi_{\mathrm{L}}$ of $1^{\circ}$ reveals no misalignment which leads to a value of $1^{\circ}$ for $\Delta \phi_{\mathrm{L}}$. Based on the agreement of the simulated and measured cutoff angle in Fig. $6, \Delta \theta_{\mathrm{L}}$, is estimated to be $0.5^{\circ}$ which is the sampling increment for $\theta_{\mathrm{L}}$ of the experiment. Analyzing the variation of $U_{\text {Meas }}$ for changes of $\pm \Delta \theta_{\mathrm{L}}$ and $\pm \Delta \phi_{\mathrm{L}}$ yields an uncertainty $\gamma_{\text {Meas }}$ of $7 \%$.

The simulations were found to be in good agreement with the measured characteristics as illustrated in Fig. 7. Distinct features such as peaks and dips can be reproduced by the simulations. The statistical analysis revealed that $71 \%$ or more of the data exhibited no significant difference between simulation and experiment as detailed through the agreement metric $\eta$ in Table I. These results indicate that the DLL-based approach of integrating $\mathrm{PhCs}$ into MC computations is suitable for detailing light propagation in a $\mathrm{PhC}$ enhanced PET detector module. Besides the validation of the simulation techniques, the results also prove that $\mathrm{PhCs}$ exhibit a transmission behavior different from plain interfaces (see Fig. 7c). On the one hand, the PD voltage for $\theta_{\mathrm{L}}=0^{\circ}$ is up to $25 \%$ lower versus the plain surface of the calibration sample. On the other hand, there is no TIR-related cutoff at $\theta_{\mathrm{L}}=33^{\circ}$. Instead, the transmission coefficient gradually decreases to zero up to an elevation angle $\theta_{\mathrm{L}}$ of $52^{\circ}$, which corresponds to an incident angle on the sample of $\theta_{\text {Sample }}=76^{\circ}$. Larger values for $\theta_{\text {Sample }}$ could not be studied due to the finite size of the PhC sample and the limited active area of the PD. The complete transmission data of sample $\mathrm{H} 1$ is depicted in Fig. 7d and shows the agreement between measurements and simulations for the entire $\phi_{\mathrm{L}^{-}}$ range. Also, the $60^{\circ}$-periodic characteristic of the hexagonal sample can be clearly identified.

\section{Simulation Study of the Impact of PhCs on the Performance of a State-of-the-Art PET Detector}

The results derived from the validation of the $\mathrm{PhC}$ characteristics affords optical $\mathrm{MC}$ simulations of $\mathrm{PhC}$ enhanced PET detector modules and benchmarking against the reference module which has no $\mathrm{PhC}$ coating. For the reference setup these calculations yield $3.42 \cdot 10^{6}$ scintillation photons that impinge on the LSO exit face at least once. Of these photons $1.61 \cdot 10^{6}$ are extracted at the first incidence yielding an extraction efficiency of 0.472 . The total amount of photons registered by the SiPMs is $1.02 \cdot 10^{6}$ which results in a reference light yield of 0.255 . The remaining photons are lost through absorption in the LSO bulk, the reflective wrapping, the inactive SiPM areas, or by escaping from the $8 \times 8$ array. The timing simulation yields a CRT of 244 ps.

The angular distribution shown in Fig. 8a was used for the optimization of two different $\mathrm{PhC}$ configurations, one with hexagonal symmetry $\left(\mathrm{C}_{\mathrm{H}}\right)$ and one with orthogonal symmetry $\left(\mathrm{C}_{\mathrm{O}}\right)$. This optimization also took into account the wavelengthdependent behavior of PhCs. The parameter sweeps indicate the highest extraction efficiencies for the parameters $a=368$ $\mathrm{nm}, s=199 \mathrm{~nm}, h=325 \mathrm{~nm}\left(\mathrm{C}_{\mathrm{H}}\right)$ and $a=554 \mathrm{~nm}, s=288$ $\mathrm{nm}, h=300 \mathrm{~nm}\left(\mathrm{C}_{\mathrm{O}}\right)$. The rather distinct spectral transmission characteristics of these configurations are depicted in Fig. 8b,c. The transmission coefficient of $\mathrm{C}_{\mathrm{O}}$ (see Fig. 8b) exhibits only minor changes over the spectrum whereas the characteristic of $\mathrm{C}_{\mathrm{H}}$ (see Fig. 8c) varies considerably with the wavelength. Nevertheless, the amount of extracted photons over the entire spectrum (see curves in Fig. 8a) exceeds the value of the reference setup for both configurations. The resulting extraction efficiencies at first incidence are 0.527 for $\mathrm{C}_{\mathrm{H}}$ and 0.515 for $\mathrm{C}_{\mathrm{O}}$, an increase by $12 \%$ and $9 \%$ in comparison to the reference setup.

The PET detector module was modified to obtain two new setups, one using the $\mathrm{PhC}$ coating $\mathrm{C}_{\mathrm{H}}$ at the interface $\mathrm{LSO} /$ glue, the other using $\mathrm{C}_{\mathrm{O}}$. In both cases, the PhC DLLs contained 12 LUTs, one for each wavelength of the considered LSO spectrum. The simulations of the two modules indicate a light yield of $0.314\left(\mathrm{C}_{\mathrm{H}}\right)$ and $0.313\left(\mathrm{C}_{\mathrm{O}}\right)$, an improvement by $23 \%$ versus the reference with no $\mathrm{PhC}$ coating. For both settings, the increases in light yield are larger than the improvements regarding the extraction efficiencies at first incidence due to the positive effect of the $\mathrm{PhC}$ accumulated over several recurrences of photons on the LSO exit face. This is because the setup of the detector module leads to little scattering of the scintillation light. For the reference configuration, those photons which have been reflected at their first incidence on the LSO exit face and return a second or third time tend to have a similar incident angle as before, leading to a low extraction efficiency (see Fig 8d). Unlike the reference configuration, PhCs transmit a broad spectrum of incident angles. Also, those photons which are not transmitted are scattered by the $\mathrm{PhC}$ upon their reflection, which causes broad spectra of incident angles (see Fig. 8e). This has an influence on the extraction efficiencies depicted in Fig. 8f, 

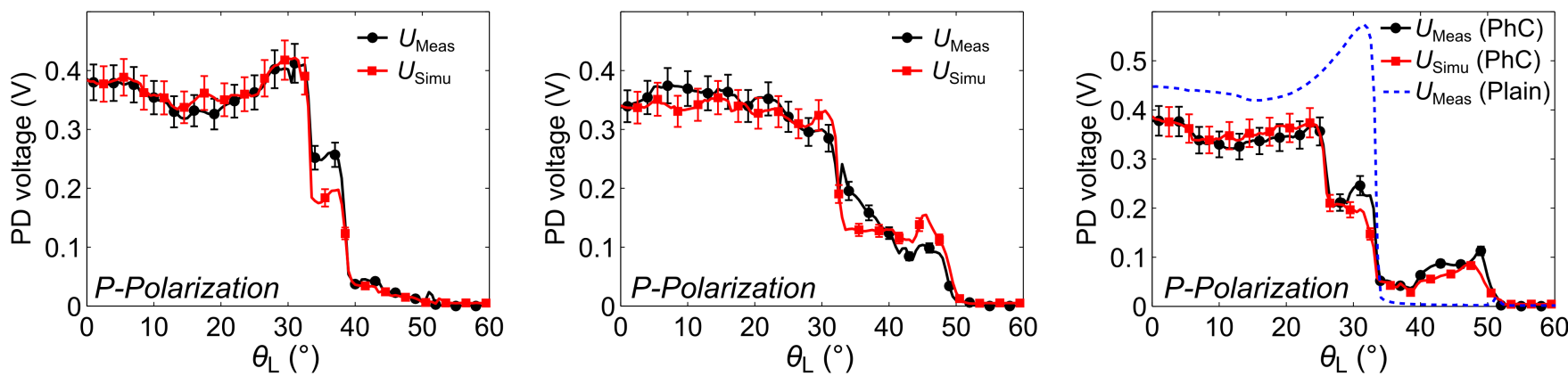

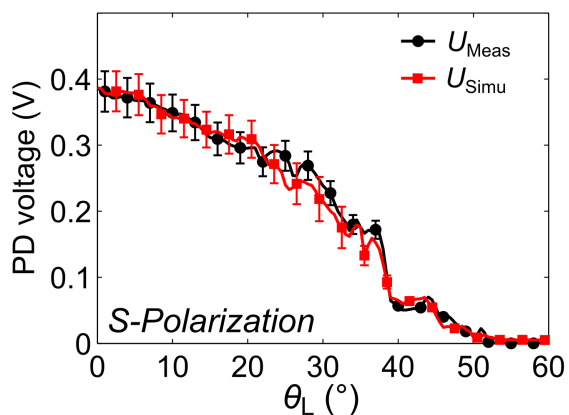

(a)
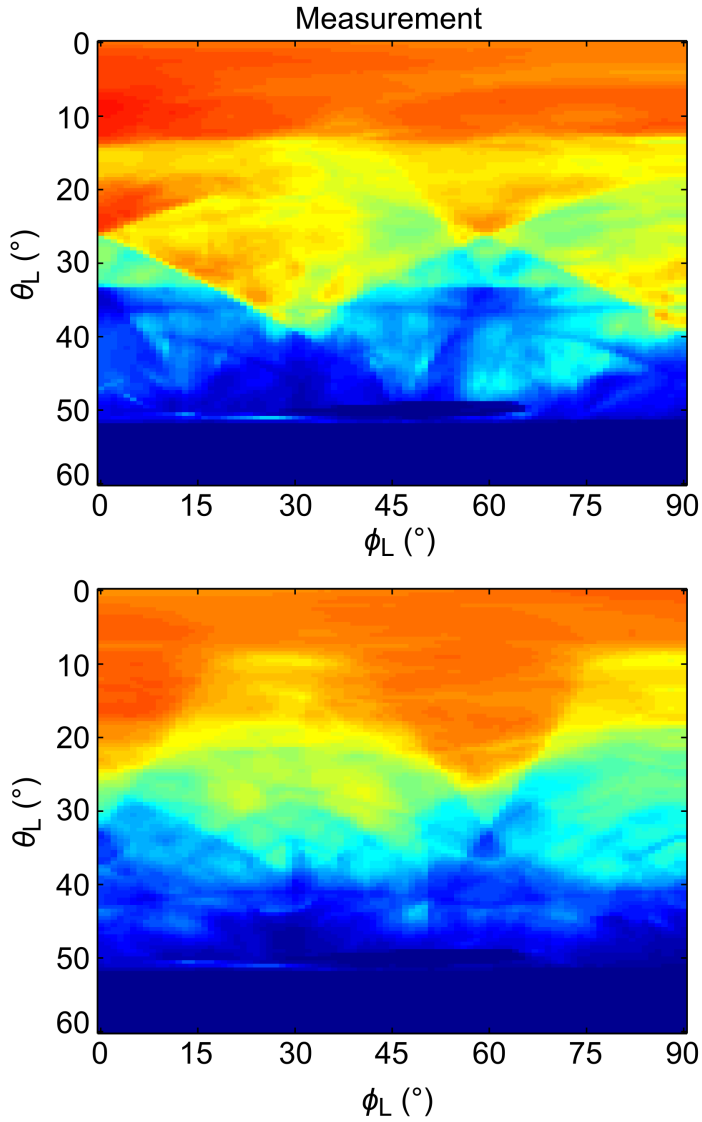

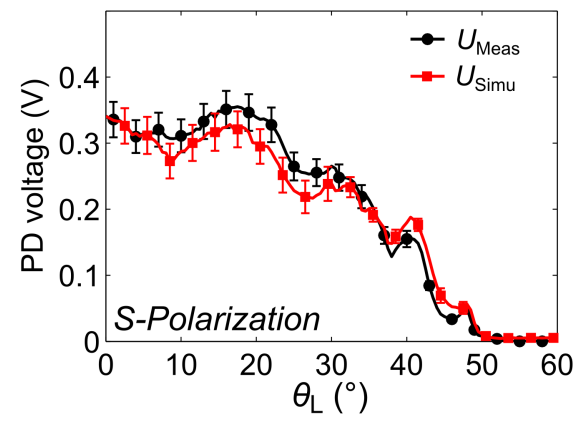

(b)

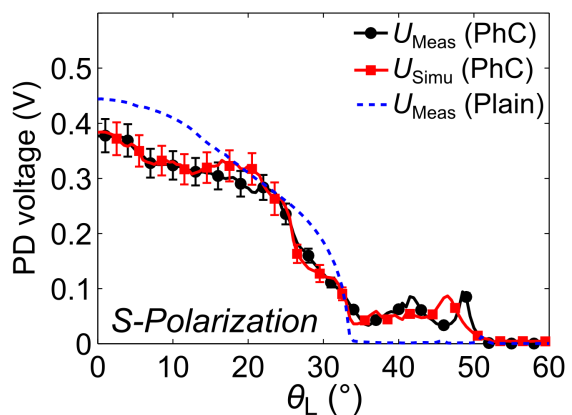

(c)
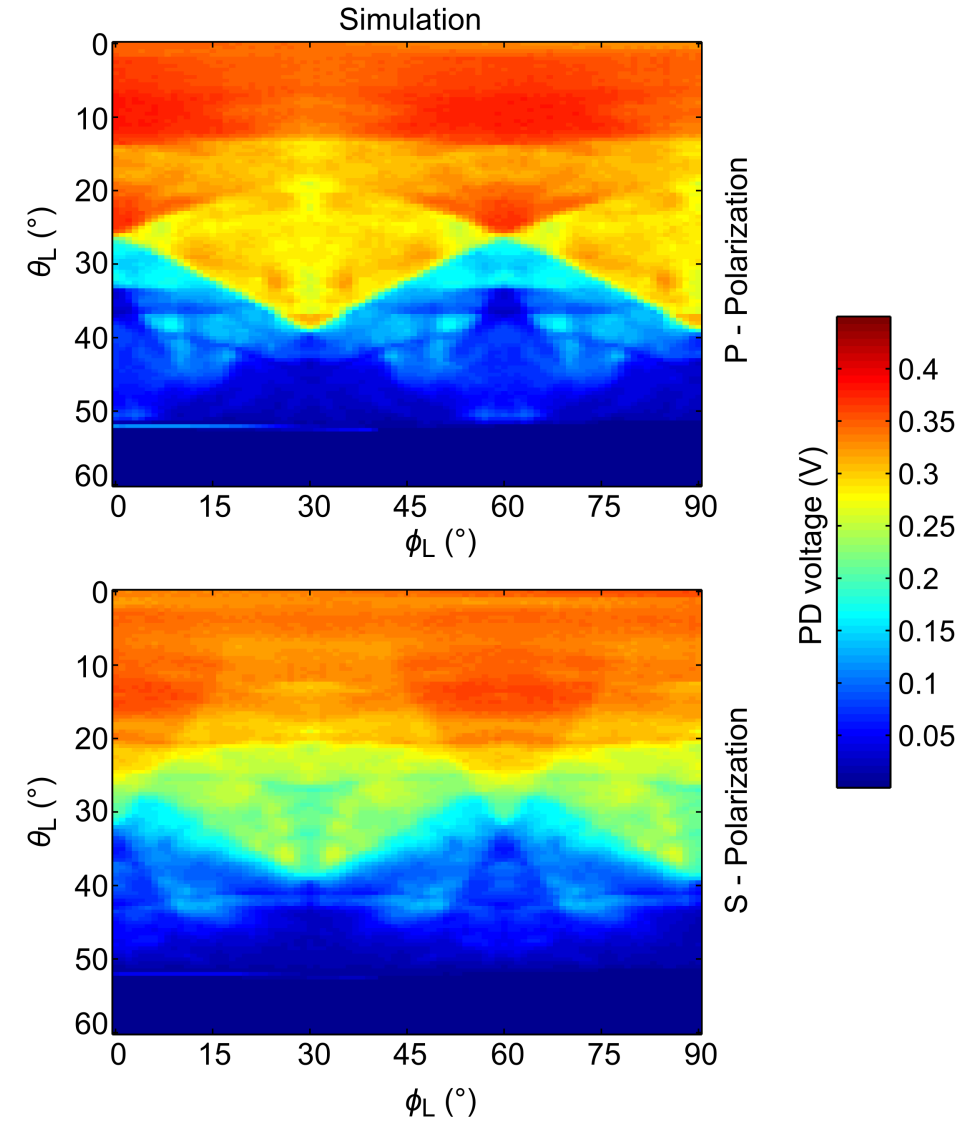

(d)

Fig. 7. Measured and simulated PD voltage versus elevation angle $\theta_{\mathrm{L}}$ for sample $\mathrm{H} 2$ at $\phi_{\mathrm{L}}=30^{\circ}$ (a), sample $\mathrm{O} 1$ at $\phi_{\mathrm{L}}=45^{\circ}$ (b), and sample $\mathrm{O} 3$ at $\phi_{\mathrm{L}}=$ $90^{\circ}$ (c) for both polarizations. The curves illustrate the agreement between experiments and simulations. In (c), the measured transmission characteristic of the plain calibration sample is added for comparison. The curves show that PhCs have a lower transmission for small values of $\theta_{\mathrm{L}}$ and do not exhibit TIR at $\theta_{\mathrm{L}}=33^{\circ}$. Error bars of simulated and measured data are displayed only at interleaved selected points for clarity. (d) PD voltage versus incident angles $\theta_{\mathrm{L}}$ and $\phi_{\mathrm{L}}$ for sample $\mathrm{H} 1$ comparing the measured and simulated data. The hexagonal symmetry of the PhC leads to a $60^{\circ}$-periodic pattern of the distribution. 


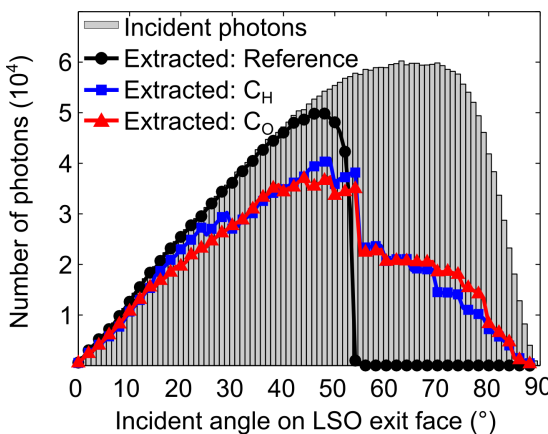

(a)

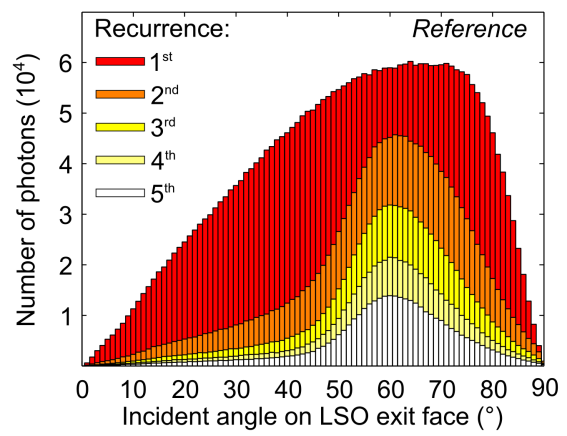

(d)

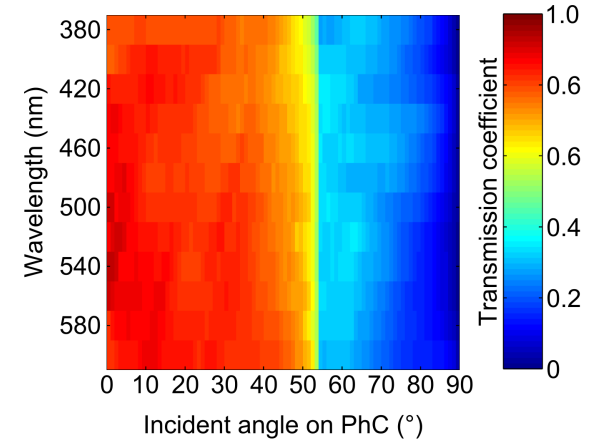

(b)

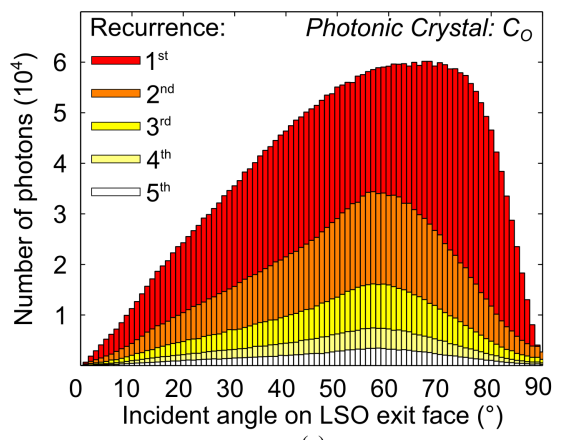

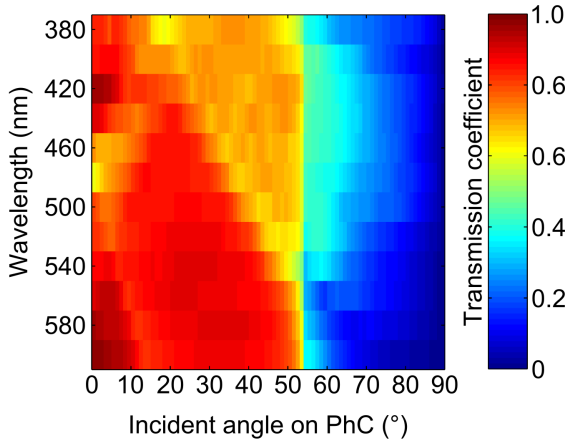

(c)

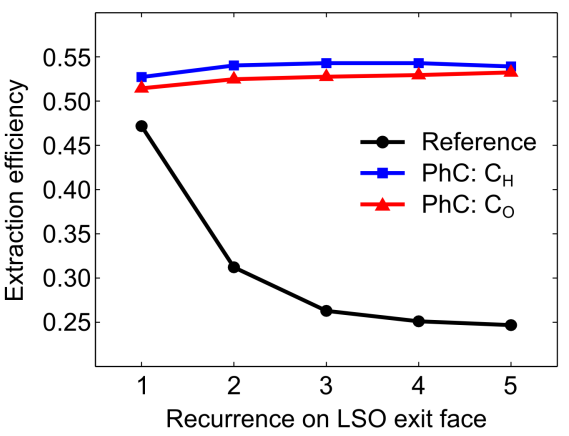

(f)

Fig. 8. (a) Angle distribution of photons at first incidence on the LSO exit face and curves of extracted photons for the 3 different configurations. Spectral transmission characteristics of PhC configuration $\mathrm{C}_{\mathrm{O}}(\mathrm{b})$ and $\mathrm{C}_{\mathrm{H}}(\mathrm{c})$. Angle distributions of photons incident on the LSO exit face over 5 recurrences for the reference (d) and the PhC configuration $\mathrm{C}_{\mathrm{O}}(\mathrm{e})$. The $\mathrm{PhC}$ interface yields improved extraction efficiencies over several recurrences (f) which causes an accumulating positive effect for the total light yield.

which are defined as the number of extracted photons at a certain recurrence divided by the total amount of incident photons at this recurrence. The fact that $\mathrm{PhC}$ configurations exhibit higher extraction efficiencies compared to the reference over several recurrences leads to an accumulating positive effect.

The propagation time distribution of detected photons is shown in Fig. 9a for the three detector setups. As observed in the optical experiments, PhC coatings lead to a reduced transmission for photons with low incident angles. Since the fastest photons mostly correspond to direct incidence on the exit face with a low angle, the propagation time distribution of the reference setup exhibits a higher peak of the leading pulse than the curves obtained for $\mathrm{C}_{\mathrm{H}}$ and $\mathrm{C}_{\mathrm{O}}$ configurations. However, approximately 240 ps after the first detected photons, the accumulated light yield for the $\mathrm{PhC}$ configurations starts to exceed the reference setup (see dashed line in Fig. 9b). The timing simulations yield CRT values of 229 ps for $\mathrm{C}_{\mathrm{H}}$ and 232 ps for $\mathrm{C}_{\mathrm{O}}$ constituting an improvement by $6 \%$ and $5 \%$, respectively. Although these values represent only modest changes, they are significantly larger than the inaccuracies of optical and timing simulations. According to the theory presented in [27], the gains in light yield obtained for configurations $\mathrm{C}_{\mathrm{H}}$ and $\mathrm{C}_{\mathrm{O}}$ should lead to an improvement of the CRT by $10 \%$. This discrepancy stems from the slightly lower leading peaks in the photon propagation time distributions of the PhC configurations compared to the reference (see Fig. 9a). This leads to only modest improvements of the CRT, which suggest that the derived $\mathrm{PhC}$ configurations do not represent optimal solutions to enhance the timing performance. This shortcoming can potentially be corrected by using an optimization procedure for the $\mathrm{PhC}$ parameters that considers not only the total light extraction, but also takes into account the photon propagation times and their impact on the CRT.

\section{CONCLUSIONS AND OUTLOOK}

This work demonstrates the implementation of photonic crystal simulations into optical MC simulations with the goal of detailing the diffractive nature of PhCs including transmission characteristics and light scattering properties. Combining photonic crystal and optical MC simulations allows modeling the impact of $\mathrm{PhC}$ coatings on the propagation of photons within complex geometries such as PET detector modules. The proposed approach was successfully validated by optical transmission measurements. The experimental results demonstrate that $\mathrm{PhC}$ coatings help to avoid a cutoff in transmission due to TIR and provide a non-zero transmission up to large incident angles. The simulation technique was used to incorporate an optimized PhC coating into a state-of-the-art PET detector module. Our results underline the potential of improving the total light yield and $\mathrm{CRT}$ by $\mathrm{PhCs}$, although the reported values are more modest than the results presented in [7], [11]. However, care must be taken for comparing these results with our study, as they were derived for individual scintillators instead of an $8 \times 8$ array and used air instead of optical glue to couple the scintillator with the photosensor. With regard to the modest gains predicted for the CRT compared to the substantial improvements in light yield by $\mathrm{PhCs}$, it is our goal 


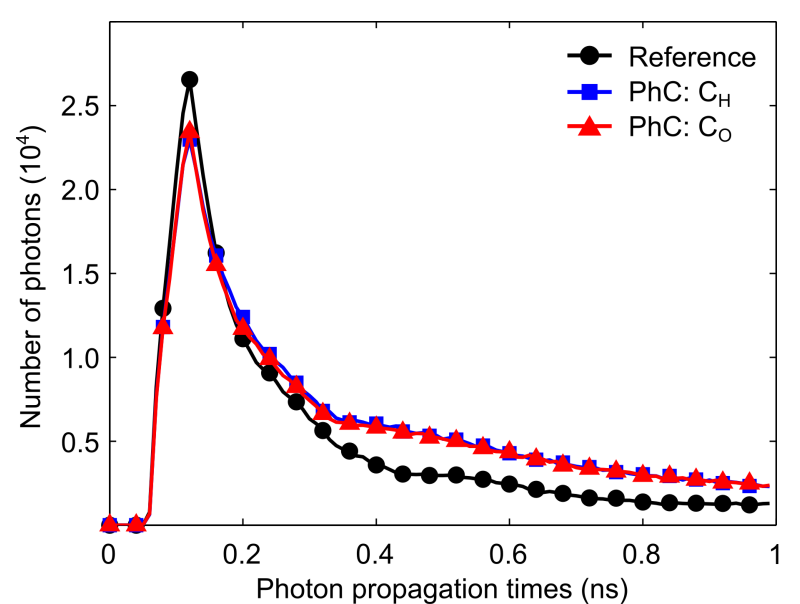

(a)

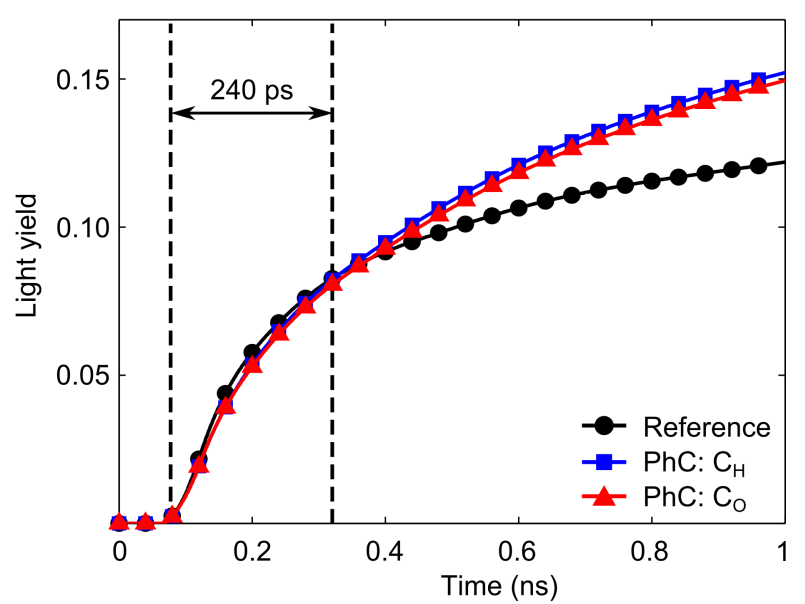

(b)

Fig. 9. (a) Propagation time distribution of detected photons for the reference setup and the two PhC configurations $\mathrm{C}_{\mathrm{H}}$ and $\mathrm{C}_{\mathrm{O}}$. (b) Accumulated light yield versus time for the same three configurations. Approximately $240 \mathrm{ps}$ after the first detected photons, the light yield of the PhC settings starts to exceed the yield of the reference.

to improve the optimization procedure to find configurations with increased timing performance. Also, the relatively low gains in CRT in relation to the high costs of using EBL for the $\mathrm{PhC}$ fabrication emphasizes the need for new production technologies that provide reduced costs and complexity. Hence, we anticipate to extend our studies to alternative fabrication procedures with the ultimate goal to provide a technique that is compatible with potential implementations in commercial PET detectors. To summarize, the proposed approach for implementing $\mathrm{PhCs}$ into an optical MC code is not restricted to a specific detector configuration and rather represents a versatile tool for modeling diffractive elements within the framework of suitable ray tracing software.

\section{ACKNOWLEDGMENT}

The authors would like to thank Dr. K. Steidel and Dr. C. Hohle from the Fraunhofer Center Nanoelectronic Technologies (IPMS-CNT, Dresden, Germany) for providing the electron beam lithography and valuable feedback for the sample fabrication process.

\section{REFERENCES}

[1] H. Rothfuss, M. Casey, M. Conti, N. Doshi, L. Eriksson, and M. Schmand, "Monte carlo simulation study of LSO crystals," IEEE Trans. Nucl. Sci., vol. 51, no. 3, pp. 770-774, Jun. 2004.

[2] E. Lorincz, G. Erdei, I. Peczeli, C. Steinbach, F. Ujhelyi, and T. Bukki, "Light output analyzes of scintillator crystal pins and array for PET detector modules," in IEEE Nuclear Science Symposium Conference Record (NSS/MIC), Oct. 2008, pp. 4868-4871.

[3] M. Janecek and W. W. Moses, "Simulating scintillator light collection using measured optical reflectance," IEEE Trans. Nucl. Sci., vol. 57, no. 3, pp. 964-970, Jun. 2010

[4] W. W. Moses and M. Ullisch, "Factors influencing timing resolution in a commercial LSO PET camera," IEEE Trans. Nucl. Sci., vol. 53, no. 1, pp. 78-85, Feb. 2006.

[5] Y. Shao, "A new timing model for calculating the intrinsic timing resolution of a scintillator detector," Phys. Med. Biol., vol. 52, no. 4, p. 1103 , Feb. 2007.

[6] A. Knapitsch, E. Auffray, C. W. Fabjan, J.-L. Leclercq, X. Letartre, R. Mazurczyk, and P. Lecoq, "Effects of photonic crystals on the light output of heavy inorganic scintillators," IEEE Trans. Nucl. Sci., vol. 60, no. 3, pp. 2322-2329, Jun. 2013.
[7] P. Lecoq, E. Auffray, and A. Knapitsch, "How photonic crystals can improve the timing resolution of scintillators," IEEE Trans. Nucl. Sci. vol. 60, no. 3, p. 1653, Jun. 2013.

[8] M. Kronberger, E. Auffray, and P. Lecoq, "Improving light extraction from heavy inorganic scintillators by photonic crystals," IEEE Trans. Nucl. Sci., vol. 57, no. 5, pp. 2475-2482, Oct. 2010.

[9] S. Fan, P. Villeneuve, J. Joannopoulos, and E. Schubert, "High extraction efficiency of spontaneous emission from slabs of photonic crystals," Phys. Rev. Lett., vol. 78, no. 17, pp. 3294-3297, Apr. 1997.

[10] J. D. Joannopoulos, S. G. Johnson, J. N. Winn, and R. D. Meade, Photonic crystals: molding the flow of light. Princetion, NJ: Princeton university press, 2011.

[11] A. Knapitsch, E. Auffray, C. W. Fabjan, J.-L. Leclercq, X. Letartre, R. Mazurczyk, and P. Lecoq, "Results of photonic crystal enhanced light extraction on heavy inorganic scintillators," IEEE Trans. Nucl. Sci., vol. 59, no. 5, pp. 2334-2339, Oct. 2012.

[12] F. Bauer, J. Corbeil, M. Schmand, and D. Henseler, "Measurements and ray-tracing simulations of light spread in LSO crystals," IEEE Trans. Nucl. Sci., vol. 56, no. 5, pp. 2566-2573, Oct. 2009.

[13] M. Moharam and T. Gaylord, "Rigorous coupled-wave analysis of planar-grating diffraction," J. Opt. Soc. Amer, vol. 71, no. 7, pp. 811$818,1981$.

[14] K. C. Johnson. (2008) Grating diffraction calculator (GD-Calc) coupled-wave theory for biperiodic diffraction gratings. [Online]. Available: http://software.kjinnovation.com/GD-Calc.pdf

[15] F. J. Massey Jr, "The Kolmogorov-Smirnov test for goodness of fit," $J$. Am. Stat. Assoc., vol. 46, no. 253, pp. 68-78, 1951.

[16] C. M. Pepin, P. Bérard, A.-L. Perrot, C. Pépin, D. Houde, R. Lecomte, C. L. Melcher, and H. Dautet, "Properties of LYSO and recent LSO scintillators for phoswich PET detectors," IEEE Trans. Nucl. Sci., vol. 51, no. 3, pp. 789-795, Jun. 2004.

[17] D. Henseler, R. Grazioso, N. Zhang, and M. Schmand, "SiPM performance in PET applications: An experimental and theoretical analysis," in IEEE Nuclear Science Symposium Conference Record (NSS/MIC), Oct. 2009, pp. 1941-1948.

[18] A. Del Guerra, N. Belcari, M. Giuseppina Bisogni, G. LLosa, S. Marcatili, G. Ambrosi, F. Corsi, C. Marzocca, G. Dalla Betta, and C. Piemonte, "Advantages and pitfalls of the silicon photomultiplier (SiPM) as photodetector for the next generation of PET scanners," Nucl. Instr. Meth. Phys. Res., Sect. A, vol. 617, no. 1-3, pp. 223-226, May 2010.

[19] (2014, 01) Filmetrics refractive index database. [Online]. Available: http://www.filmetrics.com/refractive-index-database/Si/Silicon

[20] (2014, 01) Hamamatsu Multi Pixel Photon Counters. [Online]. Available: http://www.hamamatsu.com/jp/en/product/category/ 3100/4004/index.html

[21] G. Delso, S. Fürst, B. Jakoby, R. Ladebeck, C. Ganter, S. G. Nekolla, M. Schwaiger, and S. I. Ziegler, "Performance measurements of the Siemens mMR integrated whole-body PET/MR scanner," J. Nucl. Med., vol. 52, no. 12, pp. 1914-1922, Dec. 2011. 
[22] H. Zaidi and A. Del Guerra, "An outlook on future design of hybrid PET/MRI systems," Med. Phys., vol. 38, no. 10, p. 5667, Sep. 2011.

[23] S. Agostinelli, J. Allison, K. e. Amako, J. Apostolakis, H. Araujo, P. Arce, M. Asai, D. Axen, S. Banerjee, G. Barrand et al., "GEANT4 - a simulation toolkit," Nucl. Instr. Meth. Phys. Res., Sect. A, vol. 506, no. 3, pp. 250-303, Jul. 2003.

[24] J. Breuer, S. Cho, M. Aykac, R. Grazioso, and M. Schmand, "Detailed monte-carlo simulation of SiPM-based scintillation detectors for PET," presented at the International Workshop on New Photon-detectors (PhotoDet), Orsay, France, Jun. 13-15 2012.

[25] P. Lecoq, E. Auffray, S. Gundacker, H. Hillemanns, P. Jarron, A. Knapitsch, J. Leclercq, X. Letartre, T. Meyer, K. Pauwels et al., "Progress on photonic crystals," in IEEE Nuclear Science Symposium Conference Record (NSS/MIC), Nov. 2010, pp. 1970-1975.

[26] Brewer Science high-refractive index materials. [Online]. Available: http://www.brewerscience.com/products/high-ir-materials

[27] J. B. Birks, The theory and practice of scintillation counting. Oxford, U.K.: Pergamon, 1964. 